\title{
A note on characterizing Hermitian curves via Baer sublines
}

\author{
Bart De Bruyn
}

March 9, 2015

\begin{abstract}
We consider two families of point sets in (not necessarily finite) projective planes, one of which consists of the Hermitian curves, and give a common characterization of the point sets in both families. One of the properties we use to characterize them will be the existence of a certain configuration of Baer sublines.
\end{abstract}

Keywords: Hermitian curve, projective plane, Baer subline, unital MSC2010: 51E20, 05B25

\section{Introduction}

A unital of the finite projective plane $\mathrm{PG}\left(2, q^{2}\right)$ (with $q$ a prime power) is a set of $q^{3}+1$ points meeting each line in either 1 or $q+1$ points. A line intersecting the unital in precisely $q+1$ points is called a secant line, and such a secant line is called a Baer secant line if the intersection is a Baer subline. The standard examples of unitals are the Hermitian curves (also called classical unitals), and these have the property that all secant lines are Baer secant lines. Lefèvre-Percsy [7] and Faina-Korchmáros [6] proved that this property is sufficient to characterize Hermitian curves. They showed that every unital for which all secant lines are Baer secant lines necessarily is classical. A natural question which then arises is to ask how many Baer secant lines are really necessary to conclude that a unital must be classical. In the literature, one can find several results in this direction. The original results of $[6,7]$ were improved by Barwick [2]. The best result in the literature however seems to be the result which states that a unital $\mathcal{U}$ in $\operatorname{PG}\left(2, q^{2}\right)$ is classical as soon as there exists a point $x \in \mathcal{U}$ through which all secant lines are Baer secant lines and for which there exists one additional Baer secant line (not containing $x$ ). The proof of the latter result was established in the papers $[3,5,8]$ and uses a deep result of Brown [4] regarding ovoids in the projective space $\mathrm{PG}(3, q)$. Important to mention is also the characterization result of Ball, Blokhuis and O'Keefe [1] which states that if $q$ is a prime, then a unital in $\operatorname{PG}\left(2, q^{2}\right)$ is classical as soon as there are $\left(q^{2}-2\right) q$ Baer secant lines. 
In the present paper, we give a characterization of Hermitian curves as certain sets of points that contain sufficiently many Baer secant lines (see condition (U1) below). We do no longer require in advance that the set is a unital as the earlier-mentioned characterization results do, but instead we require another condition (see condition (U3) below). The obtained characterization result will moreover be valid in the infinite case. In fact, the properties (U1), (U2) and (U3) below not only allow to characterize Hermitian curves but also the members of another family of point sets.

\section{The main result}

Let $V$ be a 3-dimensional vector space over a field $\mathbb{F}$, and denote by $\mathrm{PG}(V)$ the projective plane associated with $V$.

Suppose $X$ is a set of points of $\mathrm{PG}(V)$. A line $L$ of $\mathrm{PG}(V)$ is called an exterior line if $L \cap X=\emptyset$, a tangent line if $|L \cap X|=1$ and a secant line if $|L \cap X| \geq 2$.

Suppose $\mathbb{K}$ is a subfield of index 2 of $\mathbb{F}$. If $\bar{v}_{1}$ and $\bar{v}_{2}$ are two linearly independent vectors of $V$, then the set of all points of the form $\left\langle\lambda_{1} \bar{v}_{1}+\lambda_{2} \bar{v}_{2}\right\rangle,\left(\lambda_{1}, \lambda_{2}\right) \in(\mathbb{K} \times \mathbb{K}) \backslash\{(0,0)\}$ is called a Baer- $\mathbb{K}$-subline of $\mathrm{PG}(V)$. If $\bar{v}_{1}, \bar{v}_{2}$ and $\bar{v}_{3}$ are three linearly independent vectors of $V$, then the set of all points of the form $\left\langle\lambda_{1} \bar{v}_{1}+\lambda_{2} \bar{v}_{2}+\lambda_{3} \bar{v}_{3}\right\rangle,\left(\lambda_{1}, \lambda_{2}, \lambda_{3}\right) \in$ $(\mathbb{K} \times \mathbb{K} \times \mathbb{K}) \backslash\{(0,0,0)\}$ is called a Baer- $\mathbb{K}$-subplane of $\mathrm{PG}(V)$.

Suppose $\mathbb{K}$ is a subfield of $\mathbb{F}$ such that $\mathbb{F}$ is a separable quadratic extension of $\mathbb{K}$, and denote by $\psi$ the unique nontrivial automorphism of $\mathbb{F}$ fixing $\mathbb{K}$ elementwise. We call a set of points of $\mathrm{PG}(V)$ a $\mathbb{K}$-Hermitian curve if it has equation $X_{0}^{\psi+1}+X_{1} X_{2}^{\psi}+X_{2} X_{1}^{\psi}=0$ with respect to a certain reference system. Every point of a $\mathbb{K}$-Hermitian curve is contained in a unique tangent line. If $\mathcal{U}$ is a $\mathbb{K}$-Hermitian curve and $L$ a line of $\operatorname{PG}(V)$, then $L \cap \mathcal{U}$ is either empty, a singleton or a Baer- $\mathbb{K}$-subline. Moreover, if $L \cap \mathcal{U}$ is a Baer- $\mathbb{K}$-subline, then there exists a unique point $l$ such that $l x$ is a tangent line for every $x \in L \cap \mathcal{U}$. In fact, $l=L^{\beta}$ where $\beta$ is the unitary polarity associated with $\mathcal{U}$.

Suppose again that $\mathbb{K}$ is a subfield of index 2 of $\mathbb{F}$. If $X$ is a set of points of $\operatorname{PG}(V)$ and $x_{1}, x_{2}$ are two distinct points of $X$, then we say that $X$ satisfies Property (*) with respect to $\left(\mathbb{K}, x_{1}, x_{2}\right)$ if the following conditions are satisfied:

(U1) Any secant line through $x_{1}$ or $x_{2}$ intersects $X$ in a Baer- $\mathbb{K}$-subline.

(U2) $X \backslash x_{1} x_{2} \neq \emptyset$.

(U3) There exists a point $l \in \mathrm{PG}(V) \backslash x_{1} x_{2}$ such that $l x$ is a tangent line for every point $x \in x_{1} x_{2} \cap X$.

We say that $X$ satisfies Property (*) with respect to $\mathbb{K}$ if there exist two distinct points $x_{1}, x_{2} \in X$ such that $X$ satisfies Property $(*)$ with respect to $\left(\mathbb{K}, x_{1}, x_{2}\right)$. We will prove the following result.

Theorem 2.1 (1) If $\mathbb{F}$ is a separable quadratic extension of the field $\mathbb{K}$, then the sets of points of $\mathrm{PG}(V)$ satisfying Property $(*)$ with respect to $\mathbb{K}$ are precisely the $\mathbb{K}$ Hermitian curves of $\mathrm{PG}(V)$. 
(2) If $\mathbb{F}$ is an inseparable quadratic extension of the field $\mathbb{K}$, then the sets of points of $\mathrm{PG}(V)$ satisfying Property (*) with respect to $\mathbb{K}$ are precisely the sets of points described by a condition of the form " $\lambda X_{0}^{2}+X_{1} X_{2} \in \mathbb{K}$ ", where $\lambda \in \mathbb{F} \backslash \mathbb{K}$ and $\left(X_{0}, X_{1}, X_{2}\right)$ denote the homogeneous coordinates with respect to a fixed reference system.

Note that if $\mathbb{F}$ is an inseparable quadratic extension of $\mathbb{K}$, then the characteristic of $\mathbb{F}$ is 2 and $\mathbb{F}^{2}:=\left\{x^{2} \mid x \in \mathbb{F}\right\} \subseteq \mathbb{K}$. This implies that the condition $\lambda X_{0}^{2}+X_{1} X_{2} \in \mathbb{K}$ is welldefined: if $\left(X_{0}, X_{1}, X_{2}\right)$ satisfies this condition, then also $\left(\lambda X_{0}, \lambda X_{1}, \lambda X_{2}\right)$ with $\lambda \in \mathbb{F}^{*}$ satisfies the condition.

In Theorem 2.1, we characterized two families of sets (among which the $\mathbb{K}$-Hermitian curves) as sets of points satisfying the three properties (U1), (U2) and (U3). With the aid of a few examples, we now show that each of these conditions is in some sense necessary.

Examples. (a) Suppose $X$ is a Baer- $\mathbb{K}$-subline, $x_{1}, x_{2}$ are two distinct points of $X$ and $l$ is any point of $\mathrm{PG}(V) \backslash x_{1} x_{2}$. Then $X$ satisfies Properties (U1) and (U3), but not Property (U2).

(b) Suppose $X$ is a Baer- $\mathbb{K}$-subplane of $\mathrm{PG}(V)$ and $x_{1}, x_{2}$ are two distinct points of $X$. Then $X$ satisfies the Properties (U1) and (U2), but not (U3). Indeed, every point of $\mathrm{PG}(V) \backslash X$ is then contained in a unique secant line.

(c) Let $\mathcal{L}$ denote a collection of lines through a point $l$ and $L$ a line not containing $l$ such that $\{x \in L \mid x l \in \mathcal{L}\}$ is a Baer- $\mathbb{K}$-subline $L^{\prime}$. Let $x_{1} \in L^{\prime}$, let $L^{\prime \prime}$ denote a Baer- $\mathbb{K}$ subline of $L$ such that $L^{\prime} \cap L^{\prime \prime}=\left\{x_{1}\right\}$ and let $x_{2} \in L^{\prime \prime} \backslash L^{\prime}$. Let $Y$ denote the set of points of $\mathrm{PG}(V)$ contained on a line of $\mathcal{L}$, and put $X:=\left(Y \backslash\left(l x_{1} \cup L^{\prime}\right)\right) \cup L^{\prime \prime}$. Then $X$ satisfies Properties (U2) and (U3), but not Property (U1) if $|\mathbb{F}|>4$. However, it is still true that every secant line through $x_{1}$ intersects $X$ in a Baer- $\mathbb{K}$-subline (as well as one line through $x_{2}$, namely $x_{1} x_{2}$ ).

\section{$3(\mathbb{F}, \mathbb{K})$-sets}

Proposition 3.1 Suppose $\mathbb{F}$ is a separable quadratic extension of the field $\mathbb{K}$, and let $\psi$ denote the unique nontrivial element of $\operatorname{Gal}(\mathbb{F} / \mathbb{K})$. Let $a_{0}, a_{1}, a_{2} \in \mathbb{F}^{*}$ such that $\frac{a_{1}}{a_{2}} \notin \mathbb{K}$. Then the $\mathbb{K}$-Hermitian curve $\mathcal{U}$ of $\mathrm{PG}(V)$ having equation

$$
\frac{a_{2} a_{1}^{\psi}-a_{1} a_{2}^{\psi}}{a_{0}^{\psi+1}} X_{0}^{\psi+1}+X_{1} X_{2}^{\psi}-X_{2} X_{1}^{\psi}=0
$$

with respect to some ordered basis $\left(\bar{e}_{0}, \bar{e}_{1}, \bar{e}_{2}\right)$ of $V$ consists of all points of the form

$$
\left\langle\lambda_{1} \bar{e}_{1}+\lambda_{2} \bar{e}_{2}\right\rangle
$$

where $\left(\lambda_{1}, \lambda_{2}\right) \in(\mathbb{K} \times \mathbb{K}) \backslash\{(0,0)\}$, and all points of the form

$$
\left\langle a_{0} \bar{e}_{0}+\left(\lambda_{1} a_{1}+\lambda_{2} a_{2}\right) \bar{e}_{1}+\left(\mu_{1} a_{1}+\mu_{2} a_{2}\right) \bar{e}_{2}\right\rangle,
$$


where $\lambda_{1}, \lambda_{2}, \mu_{1}, \mu_{2} \in \mathbb{K}$ with $\left|\begin{array}{cc}\lambda_{1} & \lambda_{2} \\ \mu_{1} & \mu_{2}\end{array}\right|=1$.

Proof. Let $p=\left(X_{0}, X_{1}, X_{2}\right)$ be a point of $\mathrm{PG}(V)$.

If $X_{0}=0$, then $p \in \mathcal{U}$ if and only if $X_{1} X_{2}^{\psi}-X_{2} X_{1}^{\psi}=0$, i.e. if and only if $p$ is of the form $\left\langle\lambda_{1} \bar{e}_{1}+\lambda_{2} \bar{e}_{2}\right\rangle$, where $\left(\lambda_{1}, \lambda_{2}\right) \in(\mathbb{K} \times \mathbb{K}) \backslash\{(0,0)\}$.

If $X_{0} \neq 0$, then we may suppose that $X_{0}=a_{0}$. Since $a_{1}, a_{2}$ are linearly independent over $\mathbb{K}$, we have $X_{1}=\lambda_{1} a_{1}+\lambda_{2} a_{2}$ and $X_{2}=\mu_{1} a_{1}+\mu_{2} a_{2}$ for certain $\lambda_{1}, \lambda_{2}, \mu_{1}, \mu_{2} \in \mathbb{K}$. The point $p$ belongs to $\mathcal{U}$ if and only if

$$
\begin{gathered}
-\left(a_{1} a_{2}^{\psi}-a_{2} a_{1}^{\psi}\right)+\left(\lambda_{1} a_{1}+\lambda_{2} a_{2}\right)\left(\mu_{1} a_{1}^{\psi}+\mu_{2} a_{2}^{\psi}\right)-\left(\mu_{1} a_{1}+\mu_{2} a_{2}\right)\left(\lambda_{1} a_{1}^{\psi}+\lambda_{2} a_{2}^{\psi}\right) \\
=\left(\lambda_{1} \mu_{2}-\mu_{1} \lambda_{2}-1\right) \cdot\left(a_{1} a_{2}^{\psi}-a_{2} a_{1}^{\psi}\right)=0 .
\end{gathered}
$$

Since $a_{1} a_{2}^{\psi}-a_{2} a_{1}^{\psi} \neq 0$, this is equivalent with demanding that $\left|\begin{array}{ll}\lambda_{1} & \lambda_{2} \\ \mu_{1} & \mu_{2}\end{array}\right|=1$.

Remark. The $\mathbb{K}$-Hermitian curves of $\mathrm{PG}(V)$ are also the sets of points described by equations of the form $\lambda X_{0}^{\psi+1}+X_{1} X_{2}^{\psi}-X_{2} X_{1}^{\psi}=0$, where $\lambda \in \mathbb{F}^{*}$ such that $\lambda^{\psi}=-\lambda$. If $a_{0}, a_{1}, a_{2} \in \mathbb{F}^{*}$ with $\frac{a_{1}}{a_{2}} \notin \mathbb{K}$, then $\lambda:=\frac{a_{2} a_{1}^{\psi}-a_{1} a_{2}^{\psi}}{a_{0}^{\psi+1}}$ satisfies $\lambda \neq 0$ and $\lambda^{\psi}=-\lambda$. Conversely, every $\lambda \in \mathbb{F}^{*}$ for which $\lambda^{\psi}=-\lambda$ is of the form $\frac{a_{2} a_{1}^{\psi}-a_{1} a_{2}^{\psi}}{a_{0}^{\psi+1}}$ for certain $a_{0}, a_{1}, a_{2} \in \mathbb{F}^{*}$ with $\frac{a_{1}}{a_{2}} \notin \mathbb{K}$. Indeed, take $a_{0}=a_{1}=1$ and $a_{2}=\frac{\lambda \eta}{\eta-\eta^{\psi}}$ where $\eta \in \mathbb{F} \backslash \mathbb{K}$.

Proposition 3.2 Suppose $\mathbb{F}$ is an inseparable quadratic extension of the field $\mathbb{K}$. Let $a_{0}, a_{1}, a_{2} \in \mathbb{F}^{*}$ such that $\frac{a_{1}}{a_{2}} \notin \mathbb{K}$. Then the set $X$ of points described by the condition

$$
\frac{a_{1} a_{2}}{a_{0}^{2}} X_{0}^{2}+X_{1} X_{2} \in \mathbb{K}
$$

where $\left(X_{0}, X_{1}, X_{2}\right)$ denote the homogeneous coordinates with respect to some ordered basis $\left(\bar{e}_{0}, \bar{e}_{1}, \bar{e}_{2}\right)$ of $V$, consists of all points of the form

$$
\left\langle\lambda_{1} \bar{e}_{1}+\lambda_{2} \bar{e}_{2}\right\rangle
$$

where $\left(\lambda_{1}, \lambda_{2}\right) \in(\mathbb{K} \times \mathbb{K}) \backslash\{(0,0)\}$, and all points of the form

$$
\left\langle a_{0} \bar{e}_{0}+\left(\lambda_{1} a_{1}+\lambda_{2} a_{2}\right) \bar{e}_{1}+\left(\mu_{1} a_{1}+\mu_{2} a_{2}\right) \bar{e}_{2}\right\rangle,
$$

where $\lambda_{1}, \lambda_{2}, \mu_{1}, \mu_{2} \in \mathbb{K}$ with $\left|\begin{array}{ll}\lambda_{1} & \lambda_{2} \\ \mu_{1} & \mu_{2}\end{array}\right|=1$.

Proof. Let $p=\left(X_{0}, X_{1}, X_{2}\right)$ be an arbitrary point of $\mathrm{PG}(V)$.

If $X_{0}=0$, then $p \in X$ if and only if $X_{1} X_{2} \in \mathbb{K}$. If $X_{2} \neq 0$, then since $\mathbb{F}^{2} \subseteq \mathbb{K}$, the condition $X_{1} X_{2} \in \mathbb{K}$ is equivalent with $\frac{X_{1}}{X_{2}} \in \mathbb{K}$. So, if $X_{0}=0$, then $p \in X$ if and only if $p$ is of the form $\left\langle\lambda_{1} \bar{e}_{1}+\lambda_{2} \bar{e}_{2}\right\rangle$, where $\left(\lambda_{1}, \lambda_{2}\right) \in(\mathbb{K} \times \mathbb{K}) \backslash\{(0,0)\}$. 
If $X_{0} \neq 0$, then we may suppose that $X_{0}=a_{0}$. Since $a_{1}, a_{2}$ are linearly independent over $\mathbb{K}$, we have $X_{1}=\lambda_{1} a_{1}+\lambda_{2} a_{2}$ and $X_{2}=\mu_{1} a_{1}+\mu_{2} a_{2}$ for certain $\lambda_{1}, \lambda_{2}, \mu_{1}, \mu_{2} \in \mathbb{K}$. The point $p$ belongs to $X$ if and only if

$$
\begin{gathered}
a_{1} a_{2}+\left(\lambda_{1} a_{1}+\lambda_{2} a_{2}\right)\left(\mu_{1} a_{1}+\mu_{2} a_{2}\right) \\
=\left(\lambda_{1} \mu_{1} a_{1}^{2}+\lambda_{2} \mu_{2} a_{2}^{2}\right)+a_{1} a_{2}\left(1+\lambda_{1} \mu_{2}+\lambda_{2} \mu_{1}\right) \in \mathbb{K} .
\end{gathered}
$$

Since $a_{1}^{2}, a_{2}^{2} \in \mathbb{K}$, we have $\lambda_{1} \mu_{1} a_{1}^{2}+\lambda_{2} \mu_{2} a_{2}^{2} \in \mathbb{K}$, and since $\frac{a_{1}}{a_{2}} \notin \mathbb{K}$, we also have $a_{1} a_{2} \notin \mathbb{K}$. So, the point $p$ belongs to $X$ if and only if $1+\lambda_{1} \mu_{2}+\lambda_{2} \mu_{1}=0$, i.e. if and only if

$$
\left|\begin{array}{ll}
\lambda_{1} & \lambda_{2} \\
\mu_{1} & \mu_{2}
\end{array}\right|=1 .
$$

Remark. Suppose $\mathbb{F}$ is an inseparable quadratic extension of the field $\mathbb{K}$. If $a_{0}, a_{1}, a_{2} \in \mathbb{F}^{*}$ such that $\frac{a_{1}}{a_{2}} \notin \mathbb{K}$, then $a_{0}^{2} \in \mathbb{K}^{*}$ and $a_{1} a_{2} \notin \mathbb{K}^{*}$ and hence $\frac{a_{1} a_{2}}{a_{0}^{2}} \notin \mathbb{K}$. Conversely, if $\lambda \in \mathbb{F} \backslash \mathbb{K}$, then $\lambda=\frac{a_{1} a_{2}}{a_{0}^{2}}$ for certain $a_{0}, a_{1}, a_{2} \in \mathbb{F}^{*}$ such that $\frac{a_{1}}{a_{2}} \notin \mathbb{K}$. Indeed, we can take $a_{0}=1, a_{1}=1$ and $a_{2}=\lambda$.

We introduce a common terminology for the sets of points occurring in Propositions 3.1 and 3.2. Suppose $\mathbb{K}$ is a subfield of index 2 of $\mathbb{F}$. A set $X$ of points of $\mathrm{PG}(V)$ is called an $(\mathbb{F}, \mathbb{K})$-set with respect to some line $L$ of $\mathrm{PG}(V)$ if there exist $a_{0}, a_{1}, a_{2} \in \mathbb{F}^{*}$ with $\frac{a_{1}}{a_{2}} \notin \mathbb{K}$ and an ordered basis $\left(\bar{e}_{0}, \bar{e}_{1}, \bar{e}_{2}\right)$ of $V$ such that $L=\left\langle\bar{e}_{1}, \bar{e}_{2}\right\rangle$ and $X$ consists of all points of the form $\left\langle\lambda_{1} \bar{e}_{1}+\lambda_{2} \bar{e}_{2}\right\rangle$, where $\left(\lambda_{1}, \lambda_{2}\right) \in(\mathbb{K} \times \mathbb{K}) \backslash\{(0,0)\}$, and all points of the form $\left\langle a_{0} \bar{e}_{0}+\left(\lambda_{1} a_{1}+\lambda_{2} a_{2}\right) \bar{e}_{1}+\left(\mu_{1} a_{1}+\mu_{2} a_{2}\right) \bar{e}_{2}\right\rangle$, where $\lambda_{1}, \lambda_{2}, \mu_{1}, \mu_{2} \in \mathbb{K}$ with $\left|\begin{array}{ll}\lambda_{1} & \lambda_{2} \\ \mu_{1} & \mu_{2}\end{array}\right|=1$. So, we have the following.

Corollary 3.3 (a) If $\mathbb{F}$ is a separable quadratic extension of the field $\mathbb{K}$, then the $(\mathbb{F}, \mathbb{K})$ sets are precisely the $\mathbb{K}$-Hermitian curves of $\mathrm{PG}(V)$.

(b) If $\mathbb{F}$ is an inseparable quadratic extension of the field $\mathbb{K}$, then the $(\mathbb{F}, \mathbb{K})$-sets are precisely the sets of points described by a condition of the form " $\lambda X_{0}^{2}+X_{1} X_{2} \in \mathbb{K}$ ", where $\lambda \in \mathbb{F} \backslash \mathbb{K}$ and $\left(X_{0}, X_{1}, X_{2}\right)$ denote the homogeneous coordinates of the points with respect to a fixed reference system.

We prove the following property of $(\mathbb{F}, \mathbb{K})$-sets.

Proposition 3.4 Suppose $\mathbb{F}$ is a quadratic extension of the field $\mathbb{K}$. If $X$ is an $(\mathbb{F}, \mathbb{K})$ set and $x_{1}, x_{2}$ are two distinct points of $X$, then $X$ satisfies Property $(*)$ with respect to $\left(\mathbb{K}, x_{1}, x_{2}\right)$. Moreover, every point of $X$ is contained in a unique tangent line. 
Proof. If $\mathbb{F}$ is a separable quadratic extension of $\mathbb{K}$, then $X$ is a $\mathbb{K}$-Hermitian curve, and we already know then that $X$ must satisfy Property $\left(\mathbb{K}, x_{1}, x_{2}\right)$ and that every point of $X$ is contained in a unique tangent line.

Suppose therefore that $\mathbb{F}$ is an inseparable quadratic extension of $\mathbb{K}$. Then take an ordered basis $\left(\bar{e}_{0}, \bar{e}_{1}, \bar{e}_{2}\right)$ of $V$ and a $\lambda \in \mathbb{F} \backslash \mathbb{K}$ such that $X$ is described by the condition

$$
\lambda X_{0}^{2}+X_{1} X_{2} \in \mathbb{K}
$$

Put $l:=\left\langle\bar{e}_{0}\right\rangle$. Then $l \notin X$ since $\lambda \notin \mathbb{K}$. To prove the proposition, it suffices to show the following:

$(\dagger)$ For every point $x \in X$, the line $l x$ is a tangent line and every other line through $x$ intersects $X$ in a Baer- $\mathbb{K}$-subline.

Now, consider the quadratic form $q: V \rightarrow \mathbb{F}$ defined by

$$
q\left(X_{0} \bar{e}_{0}+X_{1} \bar{e}_{1}+X_{2} \bar{e}_{2}\right)=\lambda X_{0}^{2}+X_{1} X_{2},
$$

and denote by $f: V \times V \rightarrow \mathbb{F}$ the associated bilinear form, i.e. $f(\bar{v}, \bar{w})=q(\bar{v}+\bar{w})-$ $q(\bar{v})-q(\bar{w}), \forall \bar{v}, \bar{w} \in V$. Then $f$ is a degenerate alternating bilinear form with radical $\left\langle\bar{e}_{0}\right\rangle$.

We show the validity of $(\dagger)$. Let $\bar{v} \in V$ such that $x=\langle\bar{v}\rangle$ and let $L$ be a line through $x$. Then $L$ is determined by a 2-space $\langle\bar{v}, \bar{w}\rangle$ of $V$. Every point of $L \backslash\{x\}$ has the form $\langle\mu \bar{v}+\bar{w}\rangle$ where $\mu \in \mathbb{F}$. Note that $q(\mu \bar{v}+\bar{w})=\mu^{2} \cdot q(\bar{v})+\mu \cdot f(\bar{v}, \bar{w})+q(\bar{w})$ and $\mu^{2} q(\bar{v}) \in \mathbb{K}$.

If $f(\bar{v}, \bar{w}) \neq 0$, then $\langle\mu \bar{v}+\bar{w}\rangle \in X$ if and only if $\mu$ is of the form $\frac{k-q(\bar{w})}{f(\bar{v}, \bar{w})}$ where $k \in \mathbb{K}$, and it is straightforward to verify that for such values of $\mu$, the points $\langle\bar{v}\rangle,\langle\mu \bar{v}+\bar{w}\rangle$ form a Baer- $\mathbb{K}$-subline of $L$.

If $f(\bar{v}, \bar{w})=0$, then $\langle\bar{v}, \bar{w}\rangle=\left\langle\bar{v}, \bar{e}_{0}\right\rangle$ and without loss of generality, we may suppose that $\bar{w}=\bar{e}_{0}$. Then $q(\mu \bar{v}+\bar{w})=\mu^{2} \cdot q(\bar{v})+\lambda \notin \mathbb{K}$ for all $\mu \in \mathbb{K}$. In this case, $L$ is a tangent line.

\section{Proof of Theorem 2.1}

In this section, we suppose that $\mathbb{F}$ is a quadratic extension of the field $\mathbb{K}$. We also suppose that $X$ is a set of points of $\mathrm{PG}(V)$ satisfying Property $(*)$ with respect to $\left(\mathbb{K}, x_{1}, x_{2}\right)$, where $x_{1}$ and $x_{2}$ are two distinct points of $X$. Put $L:=x_{1} x_{2}$. Then $L \cap X$ is a Baer- $\mathbb{K}$-subline of $L$. Let $l$ be a point of $\mathrm{PG}(V) \backslash L$ such that $l x$ is a tangent line for every point $x \in L \cap X$. We choose an ordered basis $\left(\bar{e}_{0}, \bar{e}_{1}, \bar{e}_{2}\right)$ of $V$ such that $l=\left\langle\bar{e}_{0}\right\rangle, x_{1}=\left\langle\bar{e}_{1}\right\rangle, x_{2}=\left\langle\bar{e}_{2}\right\rangle$ and $L \cap X$ consists of all points of the form $\left\langle\lambda_{1} \bar{e}_{1}+\lambda_{2} \bar{e}_{2}\right\rangle$, where $\left(\lambda_{1}, \lambda_{2}\right) \in(\mathbb{K} \times \mathbb{K}) \backslash\{(0,0)\}$.

Lemma 4.1 If $X \backslash L$ contains a point $y=\left\langle a_{0} \bar{e}_{0}+a_{1} \bar{e}_{1}+a_{2} \bar{e}_{2}\right\rangle$ with $a_{0}, a_{1}, a_{2} \in \mathbb{F}$, then $a_{1}, a_{2}$ are linearly independent over $\mathbb{K}$. Hence, $a_{0}, a_{1}, a_{2} \notin \mathbb{F}^{*}$ and $\frac{a_{1}}{a_{2}} \notin \mathbb{K}$.

Proof. Since $y \neq l$, we have $\left(a_{1}, a_{2}\right) \neq(0,0)$. If $a_{1}, a_{2}$ were linearly dependent over $\mathbb{K}$, then $x=\left\langle a_{1} \bar{e}_{1}+a_{2} \bar{e}_{2}\right\rangle$ were a point of $L \cap X$ and $y \in l x$, an obvious contradiction. 
Lemma 4.2 Suppose $X \backslash L$ contains the point $y=\left\langle a_{0} \bar{e}_{0}+a_{1} \bar{e}_{1}+a_{2} \bar{e}_{2}\right\rangle$. Then for all $\lambda \in \mathbb{K}$, the set $X$ also contains the points $\left\langle a_{0} \bar{e}_{0}+\left(a_{1}+\lambda a_{2}\right) \bar{e}_{1}+a_{2} \bar{e}_{2}\right\rangle$ and $\left\langle a_{0} \bar{e}_{0}+a_{1} \bar{e}_{1}+\left(a_{2}+\lambda a_{1}\right) \bar{e}_{2}\right\rangle$.

Proof. Consider the unique Baer- $\mathbb{K}$-subplane $B$ containing the Baer- $\mathbb{K}$-sublines $x_{1} y \cap X$ and $x_{1} x_{2} \cap X$. Through the point $l$, there exists a line $M$ such that $M \cap B$ is a Baer- $\mathbb{K}$ subline. As $M$ meets $L \cap X$, it is a tangent line. As $M \cap x_{1} y$ and $M \cap x_{1} x_{2}$ define two points of $X$ contained in this tangent line, we have $M \cap x_{1} y=M \cap x_{1} x_{2}$, i.e. $M=l x_{1}$. So, the unique intersection point $\left\langle a_{0} \bar{e}_{0}+a_{1} \bar{e}_{1}\right\rangle$ of the lines $l x_{1}$ and $y x_{2}$ must belong to $B$. Hence, the unique intersection point $\left\langle a_{0} \bar{e}_{0}+\left(a_{1}+\lambda a_{2}\right) \bar{e}_{1}+a_{2} \bar{e}_{2}\right\rangle$ of the line $x_{1} y$ with the unique line through $\left\langle a_{0} \bar{e}_{0}+a_{1} \bar{e}_{1}\right\rangle$ and $\left\langle\lambda \bar{e}_{1}+\bar{e}_{2}\right\rangle$ is contained in $B$ and hence also in $X$.

By symmetry, we should also have that $\left\langle a_{0} \bar{e}_{0}+a_{1} \bar{e}_{1}+\left(a_{2}+\lambda a_{1}\right) \bar{e}_{2}\right\rangle$ belongs to $X \backslash L$.

By successive application of Lemma 4.2, we find:

Corollary 4.3 Suppose $X \backslash L$ contains the point $y=\left\langle a_{0} \bar{e}_{0}+a_{1} \bar{e}_{1}+a_{2} \bar{e}_{2}\right\rangle$. Then $X \backslash L$ contains all points of the form

$$
\left\langle a_{0} \bar{e}_{0}+\left(\lambda_{1} a_{1}+\lambda_{2} a_{2}\right) \bar{e}_{1}+\left(\mu_{1} a_{1}+\mu_{2} a_{2}\right) \bar{e}_{2}\right\rangle,
$$

where $\lambda_{1}, \lambda_{2}, \mu_{1}, \mu_{2} \in \mathbb{K}$ with $\left|\begin{array}{ll}\lambda_{1} & \lambda_{2} \\ \mu_{1} & \mu_{2}\end{array}\right|=1$.

Now, let $Y$ denote the $(\mathbb{F}, \mathbb{K})$-set consisting of all points of the form $\left\langle\lambda_{1} \bar{e}_{1}+\lambda_{2} \bar{e}_{2}\right\rangle$, where $\left(\lambda_{1}, \lambda_{2}\right) \in(\mathbb{K} \times \mathbb{K}) \backslash\{(0,0)\}$, and all points of the form $\left\langle a_{0} \bar{e}_{0}+\left(\lambda_{1} a_{1}+\lambda_{2} a_{2}\right) \bar{e}_{1}+\left(\mu_{1} a_{1}+\right.\right.$ $\left.\left.\mu_{2} a_{2}\right) \bar{e}_{2}\right\rangle$, where $\lambda_{1}, \lambda_{2}, \mu_{1}, \mu_{2} \in \mathbb{K}$ with $\left|\begin{array}{ll}\lambda_{1} & \lambda_{2} \\ \mu_{1} & \mu_{2}\end{array}\right|=1$. Then $Y \subseteq X$ by Corollary 4.3.

The following proposition, in combination with Corollary 3.3 and Proposition 3.4, finishes the proof of Theorem 2.1.

Proposition 4.4 The set $X$ coincides with the $(\mathbb{F}, \mathbb{K})$-set $Y$.

Proof. Since $Y \subseteq X$, the unique line through $x_{1}$ tangent to $Y$ necessarily coincides with $l x_{1}$ and is also the unique line through $x_{1}$ tangent to $X$. Suppose $Y$ is properly contained in $X$, and denote by $x$ an arbitrary point of $X \backslash Y$. Then $x x_{1}$ is not tangent to $X$ and hence also not to $Y$. Now, $X \cap x_{1} x$ and $Y \cap x_{1} x$ are two Baer- $\mathbb{K}$-sublines of $x_{1} x$ and $Y \cap x_{1} x$ would be properly contained in $X \cap x_{1} x$ (since $x \in\left(X \cap x_{1} x\right) \backslash\left(Y \cap x_{1} x\right)$ ). This is clearly impossible. 


\section{References}

[1] S. Ball, A. Blokhuis and C. M. O'Keefe. On unitals with many Baer sublines. Des. Codes Cryptogr. 17 (1999), 237-252.

[2] S. G. Barwick. A characterization of the classical unital. Geom. Dedicata 52 (1994), $175-180$.

[3] S. G. Barwick and C. T. Quinn. Generalising a characterisation of Hermitian curves. J. Geom. 70 (2001), 1-7.

[4] M. R. Brown. Ovoids of PG(3,q), q even, with a conic section. J. London Math. Soc. (2) 62 (2000), 569-582.

[5] L. R. A. Casse, C. M. O'Keefe and T. Penttila. Characterizations of Buekenhout-Metz unitals. Geom. Dedicata 59 (1996), 29-42.

[6] G. Faina and G. Korchmáros. A graphic characterization of Hermitian curves. Combinatorics '81 (Rome, 1981), 335-342, Ann. Discrete Math. 18, North-Holland, Amsterdam - New York, 1983.

[7] C. Lefèvre-Percsy. Characterization of Hermitian curves. Arch. Math. (Basel) 39 (1982), 476-480.

[8] C. T. Quinn and L. R. A. Casse. Concerning a characterisation of Buekenhout-Metz unitals. J. Geom. 52 (1995), 159-167. 